\title{
Influence of strut structure on hemodynamic characteristics by the boundary elementary method
}

\author{
Hongmei Peng \\ Physics and Electronic Information College, \\ Inner Mongolia University for Nationalities, China
}

\begin{abstract}
In order to avoid the formation of low shear stress areas and improve the stent stability, in the current work, the boundary elementary method was applied to investigate the influence of strut structure on hemodynamic characteristics including the blood flow fields, shear stresses on a vessel wall and pressures on the struts. Results found that the struts with an oval cross section could lead to a homogenous pressure distribution and increased shear stress, which would lower the risk for restenosis after interventional therapy. Furthermore, such results would be beneficial to the structure design and optimization of stents used for the interventional therapy.

Keywords: interventional therapy, strut structure, hemodynamic characteristics, the boundary element method.

\section{Introduction}

Recently, endovascular stent graft (ESG) has been used in the therapy of the vascular disease, due to its outstanding advantages such as small trauma, less complications and rapid recovery [1-2]. However, the stent graft would lead to the protruding of the struts on blood vessel wall and cause the blood flow to be separated and reattached. Particularly, the areas of low shear stress emerged near the struts is one of the risk factors for restenosis [3]. Animal experiments and human clinical researches [4-6] found that different strut structures have different influences on the haemodynamics characteristics. Thus, it is necessary to improve the strut structures in order to avoid the formation of low shear stress areas and increase stent stability.
\end{abstract}


Considering the issues above motioned, in the present work, boundary element method was applied to analyze the blood flow fields, shear stresses on vessel wall and pressures on the struts, where the three types of intervening struts with different geometric structure (square, circle and oval cross-section) were used. The calculation results provided some theoretical significance for optimization and design of stent.

\section{Governing equations and boundary conditions}

The vessel wall was assumed to be rigid, impermeable and stationary, so a noslip boundary condition is applied. The blood was assumed to be isotropic, homogeneous, incompressible Newtonian fluid, unsteady and laminar flow. The density and dynamic viscosity are $1050 \mathrm{~kg} / \mathrm{m}^{3}$ and $3.5 \times 10^{-3} \mathrm{~Pa} \cdot \mathrm{s}$, respectively. The mean Reynolds number is 1116 . The area of blood flow is $\Omega$, and its boundary is $\Gamma$. The blood flow is governed by the Navier-Stokes (N-S) equations, which can be written as:

$$
\left\{\begin{array}{l}
\rho \frac{\partial V}{\partial t}+\rho(V \cdot \nabla) V=-\nabla P+\mu \nabla^{2} V \\
\nabla \cdot V=0
\end{array} \text { in } \Omega,\right.
$$

the boundary conditions can be written as:

$$
\left\{\begin{array}{l}
\left.\mathrm{T} \cdot \mathrm{n}\right|_{\Gamma_{T}}=T_{s} \\
\left.V\right|_{\Gamma_{v}}=V_{s}
\end{array} \text { on } \Gamma .\right.
$$

where $\Gamma_{T} \cup \Gamma_{v}=\Gamma\left(\Gamma_{T}\right.$ and $\Gamma_{v}$ are the boundaries of the vessel wall and the inlet and outlet), $V_{s}, T_{s}$ and $\mathrm{n}$ the fluid velocity, surface force and the unit normal vector on the boundary $\Gamma, \mathrm{T}(V)$ the stress tensor corresponding to $V$.

\section{Computational methods}

The hemodynamics characteristics of interventional therapy are analyzed using the boundary element method [7]. The boundary element method does not need to measure off meshes in area, but the discrete scheme on the boundaries is required. For this reason, it is unique to solve complex boundary case. The detail computation methods are described as follows.

The equivalence integral equations of the N-S equations are obtained using the weighted residual method:

$$
\begin{aligned}
C(X) v_{k}(X) & =\oint_{\Gamma} n_{i}\left(X_{0}\right) T_{i j}^{\prime}\left(W^{k}\left(X-X_{0}\right)\right)_{0} v_{j}\left(X_{0}\right) d \Gamma_{0} \\
& -\oint_{\Gamma} n_{i}\left(X_{0}\right) T_{i j}\left(V\left(X_{0}\right)\right)_{0} w_{j}^{k}\left(X-X_{0}\right) d \Gamma_{0} \\
& +\oint_{\Gamma} n_{i}\left(X_{0}\right) v_{i}\left(X_{0}\right) v_{j}\left(X_{0}\right) w_{j}^{k}\left(X-X_{0}\right) d \Gamma_{0}
\end{aligned}
$$




$$
\begin{gathered}
-\oint_{\Omega} v_{i}\left(X_{0}\right) w_{j, i_{o}}^{k}\left(X-X_{0}\right) v_{j}\left(X_{0}\right) d \Omega_{0} \\
C(X) p(X)=-\oint_{\Gamma} n_{i}\left(X_{0}\right) q^{j}\left(X-X_{0}\right) T_{i j}\left(V\left(X_{0}\right)\right)_{0} d \Gamma_{0} \\
+\oint_{\Gamma} n_{i}\left(X_{0}\right) q^{j}\left(X-X_{0}\right) v_{i}\left(X_{0}\right) v_{j}\left(X_{0}\right) d \Gamma_{0} \\
+\frac{2}{\operatorname{Re}} \oint_{\Gamma} n_{i}\left(X_{0}\right) q_{, i_{0}}^{j}\left(X-X_{0}\right) v_{j}\left(X_{0}\right) d \Gamma_{0} \\
-\oint_{\Omega} v_{i}\left(X_{0}\right) v_{j}\left(X_{0}\right) q_{, j_{0}}^{i}\left(X-X_{0}\right) d \Omega_{0}
\end{gathered}
$$

where $i, j, k=1,2, v_{k}(k=1,2)$ is the velocity, $\quad p$ is the pressure, and $n_{i}$ is the unit normal vector on the outside boundary. $W^{k}$ and $q^{k}$ are the basic solutions of N-S equations. $T_{i j}(V)$ and $T_{i j}^{\prime}\left(W^{k}\right)$ are the stress tensors corresponding to $V$ and $W^{k}$ respectively. Here, $C(X)=1$ for $X \in \Omega ; C(X)=1 / 2$ for $X \in \Gamma$. The basic solutions and stress tensors are given as:

$$
\begin{gathered}
W_{j}^{k}=-\frac{\operatorname{Re}}{4 \pi}\left[\delta_{j k} \ln \frac{1}{\left|X-X_{0}\right|}+\frac{\left(x_{j}-x_{j 0}\right)\left(x_{k}-x_{k 0}\right)}{\left|X-X_{0}\right|^{2}}\right], \\
q^{k}=-\frac{1}{2 \pi} \frac{x_{k}-x_{k 0}}{\left|X-X_{0}\right|^{2}}, \\
T_{i j}(V)=-p \delta_{i j}+\frac{1}{\operatorname{Re}}\left(v_{i, j}+v_{j, i}\right) \\
T_{i j}^{\prime}\left(W^{k}\right)=q^{k} \delta_{i j}+\frac{1}{\operatorname{Re}}\left(w_{i, j}^{k}+w_{j, i}^{k}\right) .
\end{gathered}
$$

Subdivide the boundary $\Gamma$ into small units $\Gamma_{\beta}(\beta=1,2, \cdots, N)$, in which the values of all variables are taken as constants defined at the center of the units. The discrete forms of the integral equations are written as:

$$
\begin{aligned}
C_{\alpha} v_{k \alpha} & =\sum_{\beta=1}^{N} \sum_{i=1}^{2} \sum_{j=1}^{2} L_{i j k \alpha \beta} n_{i \beta} v_{j \beta}-\sum_{\beta=1}^{N} \sum_{j=1}^{2} M_{j k \alpha \beta} T_{j \beta}+\sum_{\beta=1}^{N} \sum_{i=1}^{2} \sum_{j=1}^{2} M_{j k \alpha \beta} n_{i \beta} v_{i \beta} v_{j \beta} \\
C_{\alpha} p_{\alpha} & =-\sum_{\beta=1}^{N} \sum_{j=1}^{2} R_{j \alpha \beta} T_{j \beta}+\sum_{\beta=1}^{N} \sum_{i=1}^{2} \sum_{j=1}^{2} S_{i j \alpha \beta} n_{i \beta} v_{j \beta}
\end{aligned}
$$

where the subscript $\beta$ denotes the $\beta$ th unit on the boundary, and $\alpha$ denotes the $\alpha$ th point in the area or on the boundary. 
$L_{i j k \alpha \beta}, M_{j k \alpha \beta}, R_{j \alpha \beta}$ and $S_{i j \alpha \beta}$ are the integral coefficients, which are given as:

$$
\begin{aligned}
& L_{i j k \alpha \beta}=\int_{\Gamma_{\beta}} T_{i j}^{\prime}\left(W^{k}\left(X_{\alpha}-X_{0}\right)\right)_{0} \mathrm{~d} \Gamma_{0}, \\
& M_{j k \alpha \beta}=\int_{\Gamma_{\beta}} w_{j}^{k}\left(X_{\alpha}-X_{0}\right) \mathrm{d} \Gamma_{0}, \\
& R_{j \alpha \beta}=\int_{\Gamma_{\beta}} q^{j}\left(X_{\alpha}-X_{0}\right) \mathrm{d} \Gamma_{0}, \\
& S_{i j \alpha \beta}=\frac{2}{\operatorname{Re}} \int_{\Gamma_{\beta}} q_{j, i_{0}}\left(X_{\alpha}-X_{0}\right) \mathrm{d} \Gamma_{0}
\end{aligned}
$$

\section{Stent design for interventional therapy}

It is well known that the stent has an influence on the hemodynamic characteristics in the interventional therapy. Therefore, the design of stent is not only considering the better adherence and no injury to intima, but also reducing the disturbance to blood flow [8-9].

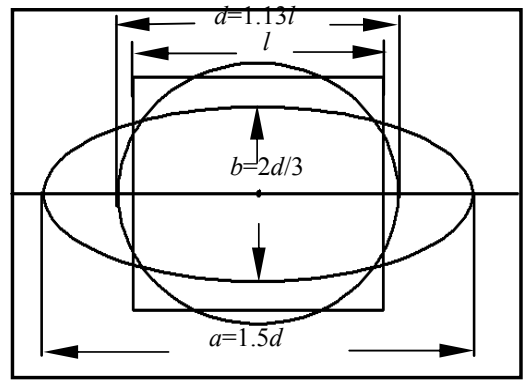

Figure 1: The cross-section view for three different struts with same cross section area.

We know that most of the traditional struts have the square or circular crosssection, as shown in Fig.1, the diameter of the circular struts should be 1.13 times of the side length of square one if both the length and the quality (or volume) of the stents are equal. In the present study, the cross-section of the strut is designed as an oval, in which the long shaft is 1.5 times of the circle diameter and the short shaft is two-thirds of it (Fig. 1). Due to a decreased thickness and no edge angle in a oval strut, the adherence is improved significantly and the risk to intimal injury is decreased. Furthermore, the vessel lumen is also much higher than those of others. Consequently, the stent with the oval cross-section strut exhibited some advantages in comparison with others.

The blood flow fields near the struts with different geometric shapes are analyzed using the boundary element method as shown in Fig. 2. In such 
analysis, the input parameters are defined based on the $l$ (the side length of square strut, it was chosen to be $1.0 \mathrm{~mm}$ ). For calculation of the blood flow fields in detail, parameters presented in Fig. 1 were used: $d=1.13 \mathrm{l}$ (diameter for the circular struts); $a=1.5 d$ (long shaft for the oval struts) and $b=2 d / 3$ (short shaft for the oval struts). The distance between the struts was designed as $3 l$. It is obviously that the low speed vortex areas appeared for the struts with square or circle cross-section as shown in Fig. 2, but it was almost invisible for the struts with oval cross-section. This result indicated that the struts with oval crosssection can significantly reduce the disturbance to the blood flow compared with those of the struts with the square or circle cross-section.

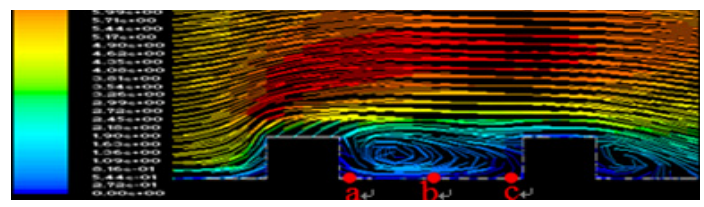

(A)

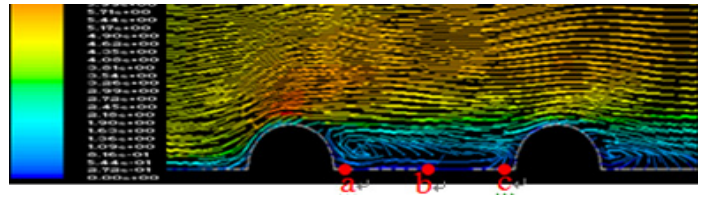

(B)

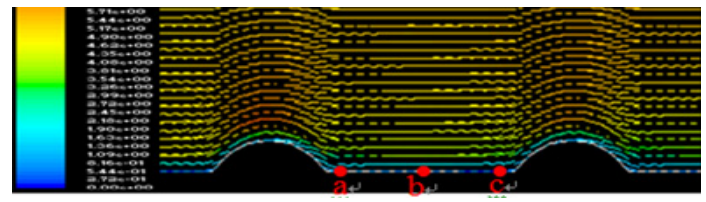

(C)

Figure 2: Flow fields near the struts with different geometric structures: (A) strut with the square cross section; (B) strut with the circular cross section; (C) strut with the oval cross section.

Fig. 3 shows the pressures distribution on the struts with different geometric shapes. It can be seen that the pressure distribution for the square and circular struts are asymmetric. And also, the pressure in upstream of the blood flow is higher than that of downstream. In this case, the stent may have a possibility to move to downstream along the blood flow. However, the pressures on the oval struts show good symmetric, thus, the stent is more stable in comparison with the former ones. 


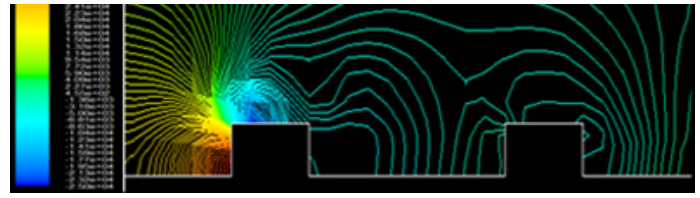

(A)

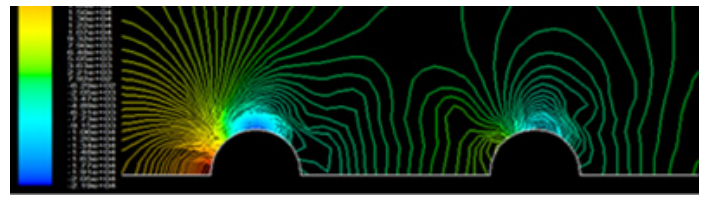

(B)

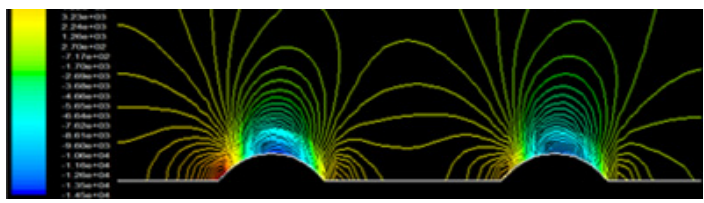

(C)

Figure 3: The pressure distributions on struts with different geometric structures: (A) strut with the square cross section; (B) strut with the circular cross section; (C) strut with the oval cross section.

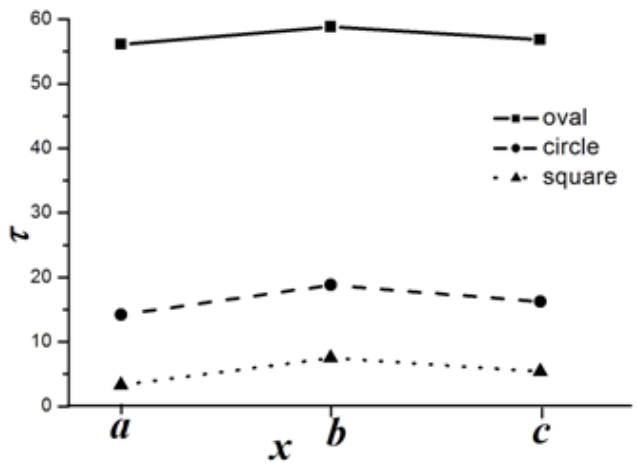

Figure 4: The comparison of shear stresses between the struts with different geometric structures.

In order to know the distribution of shear stress, the shear stress distributions at the points a, b and c (Fig. 2) were presented in Fig. 4. Clearly, the shear stress for the oval struts is largest among other cases. However, the shear stress caused by the square struts is much lower. Previous results have shown that low shear stress between the struts is a dominant factor for the cause of restenosis after 
interventional therapy. The present results show that the struts with oval crosssection have certain advantages such as homogeneous pressure distribution and increased shear stress.

\section{Conclusions}

In the current work, the boundary element method was applied to analyze the haemodynamic characteristics near the struts with different geometric shapes. The results found that the struts with the oval cross section presented some advantages, such as homogeneous pressure distribution and increased shear stress, in comparison with the struts with the square and circle cross-section. Such results would be beneficial to the structure design and optimization of stents used for the interventional therapy.

\section{References}

[1] S. Bode-Jänisch, A. Schmidt, D. Günther, M. Stuhrmann, A. Fieguth. Aortic dissecting aneurysms-Histopathological findings [J]. Forensic Science International, 2012, 214:13-17.

[2] Qiao Aike, Liu Youjun. Medical application oriented blood flow simulation [J]. Clinical Biomechanics, 2008, 23(1):S130-S136.

[3] Hong Jinxing, Wei Lan, Fu Ceji, Tan Wenchang. Blood flow and macromolecular transport in complex blood vessel [J]. Clinical Biomechanics, 2008, 23:S125-S129.

[4] Sung Wan Ham, Vincent L. Rowe, Christian Ochoa, Terry Chong. Thoracic aortic stent-grafting for acute, complicated, type B aortic dissections [J]. Annals of Vascular Surgery, 2011, 25(3):333-339.

[5] Chung-Dann Kan, Yu-Jen Yang. Double telescopic anastomosis with interrupted suture technique in acute aortic dissection [J]. The Annals of Thoracic Surgery, 2011, 91(5):1630-1631.

[6] Woong Chol Kang, Roy K. Greenberg, Tara M. Mastracci, Matthew J. Eagleton. Endovascular repair of complicated chronic distal aortic dissections: Intermediate outcomes and complications Original [J]. The Journal of Thoracic and Cardiovascular Surgery, 2011, 142(5):1074-1083.

[7] Peng Hong-mei, Yang De-que. The boundary element analysis on double bifurcation arterial hemodynamic characteristics [J]. Journal of medical Biomechanics, 2010, 25(4):283-285 (in Chinese).

[8] Martin Czerny, Sybilla Stöhr, Thierry Aymard, Gottfried H. Sodeck. Effect on false-lumen status of a combined vascular and endovascular approach for the treatment of acute type A aortic dissection [J]. European Journal of Cardio-Thoracic Surgery, 2011, 31: 342-346.

[9] Fu Wenyu, Gu Zhaoyong, Meng Xianlong, Chu Bo, Qiao Aike. Numerical simulation of hemodynamics in stented internal carotid aneurysm based on patient-specific model [J]. Journal of Biomechanics, 2010, 43(7):1337-1342. 